PROCEEDINGS OF THE

AMERICAN MATHEMATICAL SOCIETY

Volume 137, Number 9, September 2009, Pages 2905-2912

S 0002-9939(09)09861-X

Article electronically published on March 18, 2009

\title{
JACOBSON'S THEOREM FOR BILINEAR FORMS IN CHARACTERISTIC 2
}

\author{
AHMED LAGHRIBI
}

(Communicated by Bernd Ulrich)

\begin{abstract}
The aim of this paper is to extend to bilinear forms in characteristic 2 a result of Jacobson which states that over any field, two Albert quadratic forms are similar if and only if they have the same Clifford invariant.
\end{abstract}

\section{INTRODUCTION}

Throughout this paper $F$ denotes a field. To a biquaternion algebra $Q_{1} \otimes_{F} Q_{2}$ we attach a 6-dimensional quadratic form $\varphi$ given by $N_{Q_{1}} \perp-N_{Q_{2}} \simeq H \perp \varphi$, where $N_{Q_{i}}$ is the norm form of the quaternion algebra $Q_{i}$ and $H$ is the hyperbolic plane ( $\perp$ and $\simeq$ mean the orthogonal sum and isometry, respectively). The form $\varphi$ has trivial signed discriminant (resp. trivial Arf invariant) if the characteristic is $\neq 2$ (resp. if the characteristic is 2). We call such a form an Albert quadratic form. A well-known result of Jacobson states that two biquaternion algebras are isomorphic if and only if their corresponding Albert quadratic forms are similar 3 . In other words, this result says that two Albert quadratic forms are similar if and only if they have the same Clifford invariant. Using a method based on quadratic form theory, Mammone and Shapiro recovered Jacobson's result 7 and also completed it in characteristic 2 (see [7, comments in the middle of page 529]). The Clifford invariant of a quadratic form (nonsingular quadratic form if the characteristic is 2) is defined in the 2-torsion ${ }_{2} \mathrm{Br}(F)$ of the Brauer group of $F$. It is well-known that ${ }_{2} \mathrm{Br}(F)$ is isomorphic to $I^{2} F / I^{3} F\left(\right.$ resp. $\left.I_{q}^{2} F / I_{q}^{3} F\right)$ if the characteristic is not 2 (resp. if the characteristic is 2), where $I^{k} F=(I F)^{k}$ and $I_{q}^{k} F=I^{k-1} F \otimes W_{q}(F)$, with $I F$ denoting the ideal of the Witt ring of even-dimensional quadratic forms or bilinear forms according to whether the characteristic is different from or equal to 2 , and $W_{q}(F)$ denoting the Witt group of nonsingular quadratic forms [1]. These isomorphisms are due to Merkurjev [8] and Sah [10, respectively. Since in characteristic 2 we should distinguish between quadratic and bilinear forms, it is natural to ask whether an analogue of Jacobson's result holds for bilinear forms in characteristic 2. As for quadratic forms, an Albert bilinear form means a 6-dimensional form with trivial determinant. Of course there is no notion of Clifford invariant for bilinear forms in characteristic 2, but we have a result of Kato which gives an analogue of Merkurjev's and Sah's results cited above (see Theorem 2.1) and where the group of finite sums

Received by the editors June 27, 2007, and, in revised form, December 13, 2008.

2000 Mathematics Subject Classification. Primary 11E04; Secondary 11E81.

Key words and phrases. Bilinear forms, totally singular quadratic forms, norm field, norm degree, differential forms. 
of logarithmic differential forms (see below) is used in place of ${ }_{2} \mathrm{Br}(F)$ in the case of quadratic forms. We will see that this ingredient suffices to get a result similar to that of Jacobson.

Recall that, for $n \geq 1$, one denotes by $\Omega_{F}^{n}=\bigwedge^{n} \Omega_{F}^{1}$ the vector space of $n$ differential forms over $F$, where $\Omega_{F}^{1}$ is the $F$-vector space generated by symbols $d x$, $x \in F$, subject to the relations $d(x+y)=d x+d y$ and $d(x y)=x d y+y d x$, for $x, y \in F$. An element $\frac{d x_{1}}{x_{1}} \wedge \cdots \wedge \frac{d x_{n}}{x_{n}}, x_{i} \neq 0$, is called an $n$-logarithmic symbol. A sum of $n$-logarithmic symbols has length $k$ if it is a sum of $k n$-logarithmic symbols but not a sum of fewer than $k n$-logarithmic symbols.

All bilinear forms considered in this paper are supposed to be of finite dimension, symmetric and regular. For $a_{1}, \cdots, a_{n} \in F^{*}:=F \backslash\{0\}$, let $\left\langle a_{1}, \cdots, a_{n}\right\rangle_{b}$ denote the bilinear form given by the polynomial $\sum_{i=1}^{n} a_{i} x_{i} y_{i}$. If $\gamma$ is an $\mathrm{Al}$ bert bilinear form which represents a nonzero scalar, then it is diagonalizable 1, Prop. 3.5, page 13], and thus $\gamma \simeq \alpha\langle r, s, r s, u, v, u v\rangle_{b}$ for suitable scalars $\alpha, r, s, u, v \in F^{*}$. We associate to such a form $\gamma$ the following sum of 2-logarithmic symbols: $e^{2}(\gamma):=\frac{d r}{r} \wedge \frac{d s}{s}+\frac{d u}{u} \wedge \frac{d v}{v} \in \Omega_{F}^{2}$. If $\gamma$ is an Albert bilinear form which does not represent a nonzero scalar, then we put $e^{2}(\gamma)=0 \in \Omega_{F}^{2}$. The element $e^{2}(\gamma)$ is an invariant of $\gamma$ modulo $I^{3} F$ (see below). Our main result in this paper is the following theorem:

Theorem 1.1. Let $F$ be a field of characteristic 2, and let $\gamma_{1}, \gamma_{2}$ be two Albert bilinear forms. Then we have the following statements:

(1) The length of $e^{2}\left(\gamma_{1}\right)$ is 2,1 or 0 according to whether the Witt index of $\gamma_{1}$ is 0,1 or 3 .

(2) If $\gamma_{1}$ and $\gamma_{2}$ are anisotropic, then $\gamma_{1}$ is similar to $\gamma_{2}$ if and only if $e^{2}\left(\gamma_{1}\right)=$ $e^{2}\left(\gamma_{2}\right)$.

By using Theorem 2.1 of Kato, we reduce the proof of Theorem 1.1 to the use of methods from quadratic and bilinear form theory as was used in [7. However, some of our results differ from those given in [7, and their proofs require more detail. The reason is that for bilinear forms in characteristic 2 some classical results, like the Witt cancellation and the representation criterion, fail. Moreover, we will rely on the connection between totally singular quadratic forms and bilinear forms, and the notion of norm degree introduced in [2, Section 8] will play a crucial rôle.

The rest of this paper is organized as follows. We finish this section by giving some background on quadratic and bilinear forms in characteristic 2. The next section is devoted to some results needed for the proof of Theorem 1.1, and more particularly it will concern the similarity of 4-dimensional bilinear forms in characteristic 2 . Then in the third section we prove the theorem.

From now on, we assume that $F$ is of characteristic 2. To keep this paper selfcontained we briefly recall some notions. More details can be found in [1 and [2].

For a quadratic (or bilinear) form $\varphi$, we denote by $\operatorname{dim} \varphi$ its dimension. Two quadratic (or bilinear) forms $\varphi$ and $\psi$ are called similar if $\varphi \simeq \alpha \psi$ for some scalar $\alpha \in F^{*}$. A quadratic (or bilinear) form $\varphi$ is called a subform of another form $\psi$ if there exists a form $\varphi^{\prime}$ such that $\psi \simeq \varphi \perp \varphi^{\prime}$. 
To any bilinear form $B$ with underlying vector space $V$ we associate a unique quadratic form $\widetilde{B}$ given by $\widetilde{B}(v)=B(v, v)$ for $v \in V$. We say that a scalar $\alpha \in F$ is represented by $B$ if it is represented by the quadratic form $\widetilde{B}$, i.e., $\alpha=\widetilde{B}(v, v)$ for some $v \in V$. A quadratic form $\varphi$ such that $\varphi \simeq \widetilde{B}$ is called a totally singular quadratic form.

For $a_{1}, \cdots, a_{n} \in F$, let $\left\langle a_{1}, \cdots, a_{n}\right\rangle$ denote the quadratic form given by the polynomial $\sum_{i=1}^{n} a_{i} x_{i}^{2}$.

For a field extension $K / F$ and a quadratic (or bilinear) form $\varphi$, the form $\varphi \otimes K$ is denoted by $\varphi_{K}$.

For a quadratic form $\varphi$, we denote by $F(\varphi)$ its function field, i.e., the function field of the affine quadric given by $\varphi=0$. The function field of a bilinear form $B$ is by definition the field $F(\widetilde{B})$.

A quadratic form $\varphi$ with underlying vector space $V$ is isotropic if there exists $v \in V \backslash\{0\}$ such that $\varphi(v)=0$. A bilinear form $B$ is isotropic if the quadratic form $\widetilde{B}$ is isotropic. A quadratic (or bilinear) form is anisotropic if it is not isotropic.

Any bilinear form $B$ decomposes as follows: $B \simeq B_{\text {an }} \perp M_{1} \perp \cdots \perp M_{n}$, where $B_{\text {an }}$ is anisotropic and $M_{i}$ is given by the matrix $\left(\begin{array}{cc}a_{i} & 1 \\ 1 & 0\end{array}\right)$ for some $a_{i} \in F$ $(1 \leq i \leq n)$. The form $B_{\text {an }}$ is unique up to isometry [9], and we call it the anisotropic part of $B$. The integer $n$ is called the Witt index of $B$ and it is denoted by $i_{W}(B)$. The form $B$ is called metabolic if $\operatorname{dim} B=2 i_{W}(B)$. Two bilinear forms $B$ and $C$ are called equivalent, denoted by $B \sim C$, if $B \perp M \simeq C \perp M^{\prime}$ for some metabolic forms $M$ and $M^{\prime}$. By the uniqueness of the anisotropic part, the condition $B \sim C$ implies that $B_{\mathrm{an}} \simeq C_{\mathrm{an}}$. Recall that isotropy of a bilinear form $B$ is equivalent to saying that $i_{W}(B) \geq 1$.

The form $B=\left\langle 1, a_{1}\right\rangle_{b} \otimes \cdots \otimes\left\langle 1, a_{n}\right\rangle_{b}$ is called an $n$-fold bilinear Pfister form, and it is denoted by $\left\langle\left\langle a_{1}, \cdots, a_{n}\right\rangle\right\rangle_{b}$. In this case, we denote $\widetilde{B}$ by $\left\langle\left\langle a_{1}, \cdots, a_{n}\right\rangle\right\rangle$ and we call it an $n$-fold quasi-Pfister form. Recall that a bilinear Pfister form $B$ is isotropic if and only if it is metabolic and it satisfies the multiplicativity property, which means that a scalar $\alpha \in F^{*}$ is represented by $B$ if and only if $B \simeq \alpha B$. For any integer $n \geq 1$, the ideal $I^{n} F$ is additively generated by $n$-fold bilinear Pfister forms.

We say that a totally singular quadratic form $\varphi$ is a quasi-Pfister neighbor if there exists a quasi-Pfister form $\pi$ such that $\varphi$ is similar to a subform of $\pi$ and $2 \operatorname{dim} \varphi>\operatorname{dim} \pi$. In this case, $\pi$ is unique up to isometry, and for any field extension $K / F$, the forms $\varphi_{K}$ and $\pi_{K}$ are simultaneously isotropic or anisotropic.

The norm field of a nonzero totally singular quadratic form $\varphi$, denoted by $N_{F}(\varphi)$, is the field $F^{2}\left(\alpha \beta \mid \alpha, \beta \in D_{F}(\varphi)\right)$, where $D_{F}(\varphi)$ is the set of scalars in $F^{*}$ represented by $\varphi$. We denote by $\operatorname{ndeg}_{F}(\varphi)$ the integer $\left[N_{F}(\varphi): F^{2}\right]$, and we call it the norm degree of $\varphi$. It is clear that $N_{F}(\varphi)=N_{F}(\alpha \varphi)$ for any scalar $\alpha \in F^{*}$. If $\varphi$ is anisotropic and $2^{n}<\operatorname{dim} \varphi \leq 2^{n+1}$, then $\operatorname{ndeg}_{F}(\varphi) \geq 2^{n+1}$, and $\operatorname{ndeg}_{F}(\varphi)=2^{n+1}$ if and only if $\varphi$ is a quasi-Pfister neighbor. If $\varphi$ and $\psi$ are anisotropic such that $\varphi_{F(\psi)}$ is isotropic, then $\psi$ is totally singular and $N_{F}(\psi) \subset N_{F}(\varphi)$. Moreover, there is a bijection between anisotropic $n$-fold quasi-Pfister forms and purely inseparable extensions of $F^{2}$ of degree $2^{n}$ inside $F$; it is given by $F^{2}\left(a_{1}, \cdots, a_{n}\right) \leftrightarrow\left\langle\left\langle a_{1}, \cdots, a_{n}\right\rangle\right\rangle$. We refer to [2, Section 8] for more details on the norm field and some of its applications. 


\section{Preliminaries}

It is clear that the map $d: F \longrightarrow \Omega_{F}^{1}, x \mapsto d x$, extends to a map $d: \Omega_{F}^{n} \longrightarrow \Omega_{F}^{n+1}$ defined by

$$
d\left(x d x_{1} \wedge d x_{2} \wedge \cdots \wedge d x_{n}\right)=d x \wedge d x_{1} \wedge d x_{2} \wedge \cdots \wedge d x_{n} .
$$

There is a well-defined homomorphism $\wp_{n}: \Omega_{F}^{n} \longrightarrow \Omega_{F}^{n} / d \Omega_{F}^{n-1}$, given on generators by

$$
\wp_{n}\left(x \frac{d x_{1}}{x_{1}} \wedge \cdots \wedge \frac{d x_{n}}{x_{n}}\right)=\overline{\left(x^{2}-x\right) \frac{d x_{1}}{x_{1}} \wedge \cdots \wedge \frac{d x_{n}}{x_{n}} .}
$$

We write $\nu_{F}(n)$ for the kernel of this map. A crucial result that we will use is the following theorem due to Kato, which gives a link between this kernel and bilinear forms. Kato also established a relation between the cokernel of $\wp_{n}$ and quadratic forms, but we don't need it here.

Theorem 2.1 (4]). For any integer $n \geq 1$, there is an isomorphism $I^{n} F / I^{n+1} F \stackrel{\sim}{\rightarrow}$ $\nu_{F}(n)$, given by

$$
\sum\left\langle\left\langle a_{1}, \cdots, a_{n}\right\rangle\right\rangle_{b}+I^{n+1} F \mapsto \sum \frac{d a_{1}}{a_{1}} \wedge \cdots \wedge \frac{d a_{n}}{a_{n}} .
$$

We will use this theorem in the case of $n=2$. Another result that we need is the following theorem, which gives information on the dimensions of bilinear forms in $I^{n} F$ :

Theorem 2.2. Let $B \in I^{n} F$ be anisotropic $(n \geq 1)$. Then:

(1) $\operatorname{dim} B=0$ or $\operatorname{dim} B \geq 2^{n}$. Furthermore, if $\operatorname{dim} B=2^{n}$, then $B$ is similar to an $n$-fold bilinear Pfister form.

(2) If $\operatorname{dim} B>2^{n}$, then $\operatorname{dim} B \geq 2^{n}+2^{n-1}$.

Proof. (1) This is [6, Lem. 4.8].

(2) Let $F(t)$ be the rational function field in the variable $t$ over $F$, and let $\left[1, t^{-1}\right]$ be the nonsingular quadratic form $x^{2}+x y+t^{-1} y^{2}$ over $F(t)$. The nonsingular quadratic form $\varphi=B \otimes\left[1, t^{-1}\right]$ is anisotropic [6, Lem. 4.6] and belongs to $I^{n} F \otimes$ $W_{q}(F(t))$. Let $K$ be a field of characteristic 0 which is complete for a discrete valuation and whose residue field is $F(t)$. The lifting of $\varphi$ to $K$, denoted by $\varphi^{\prime}$, is an anisotropic quadratic form in $I^{n+1} K$ of dimension bigger than $2^{n+1}$. It follows from a result of Vishik [1] that $\operatorname{dim} \varphi^{\prime} \geq 2^{n+1}+2^{n}$. Hence, $\operatorname{dim} \varphi \geq 2^{n+1}+2^{n}$, i.e., $\operatorname{dim} B \geq 2^{n}+2^{n-1}$.

Lemma 2.3. Let $B$ be an anisotropic bilinear form and let $d \in F^{*} \backslash F^{* 2}$. Then, $B$ becomes metabolic over $F(\sqrt{d})$ if and only if $B \simeq \alpha_{1}\left\langle 1, d+x_{1}^{2}\right\rangle_{b} \perp \cdots \perp$ $\alpha_{n}\left\langle 1, d+x_{n}^{2}\right\rangle_{b}$ for some $\alpha_{i}, x_{i} \in F$ with $\alpha_{i} \neq 0,1 \leq i \leq n$.

Proof. By [5, Lem. 3.4], the bilinear form $B$ is metabolic over $F(\sqrt{d})$ if and only if $B \simeq C_{1} \perp \cdots \perp C_{n}(n \geq 1)$, where each bilinear form $C_{i}$ is given by a matrix $\left(\begin{array}{cc}a_{i} & b_{i} \\ b_{i} & a_{i} d\end{array}\right)$ for $a_{i}, b_{i} \in F$ and $a_{i} \neq 0$. Moreover, it is easy to check that $C_{i} \simeq$ $\alpha_{i}\left\langle 1, d+x_{i}^{2}\right\rangle_{b}$ for suitable scalars $\alpha_{i}, x_{i} \in F$ with $\alpha_{i} \neq 0$. Hence, the lemma follows. 
Some results on bilinear forms of dimension 4 will be needed, more particularly properties concerning the similarity between such forms. Before we give our contribution in this direction (Proposition 2.5 and Corollary 2.6), we start by clarifying the question of whether a 4-dimensional bilinear form becomes isotropic over the inseparable quadratic extension given by its determinant. Recall that an anisotropic quadratic form of dimension 4 (nonsingular if the characteristic is 2) stays anisotropic over the quadratic extension given by its signed discriminant (or the separable quadratic extension given by its Arf invariant). For bilinear forms in characteristic 2 the situation is different as the following proposition shows:

Proposition 2.4. Let $B$ be a bilinear form of dimension 4 whose determinant is not trivial. Then, $B$ becomes isotropic over the quadratic inseparable extension given by its determinant if and only if $\operatorname{ndeg}_{F}(\widetilde{B}) \leq 4$.

Proof. Write $B=\alpha\langle r, s, r s, d\rangle_{b}$ for suitable scalars $\alpha, r, s \in F^{*}$. One has $N_{F}(\widetilde{B})=$ $F^{2}(r, s, d)$, and thus $\operatorname{ndeg}_{F}(\widetilde{B}) \leq 8$. We may suppose that $B$ is anisotropic, in particular that $\langle r, s, r s\rangle_{b}$ is also anisotropic, and thus $\left[F^{2}(r, s): F^{2}\right]=4$. Hence, $\operatorname{ndeg}_{F}(\widetilde{B}) \in\{4,8\}$.

Suppose $\operatorname{ndeg}_{F}(\widetilde{B})=4$. Hence, $d \in F^{2}(r, s)$. Since $d \notin F^{2}$, we have $F^{2}(r, s)=$ $F^{2}(d, k)$ for some $k \in F^{*}$. In particular, $\langle\langle r, s\rangle\rangle \simeq\langle\langle d, k\rangle\rangle$. Now it is clear that $\langle\langle r, s\rangle\rangle_{F(\sqrt{d})}$ is isotropic. Then, $\langle r, s, r s, d\rangle_{F(\sqrt{d})}$ is also isotropic; i.e., $B_{F(\sqrt{d})}$ is isotropic.

Conversely, if $B_{F(\sqrt{d})}$ is isotropic, then $\left(\langle\langle r, s\rangle\rangle_{b}\right)_{F(\sqrt{d})}$ is metabolic. By Lemma 2.3 it is clear that $\langle\langle r, s\rangle\rangle_{b} \simeq\left\langle\left\langle d+x^{2}, y\right\rangle\right\rangle_{b}$ for suitable scalars $x, y \in F$ with $y \neq 0$. Hence, $d \in F^{2}(r, s)$ and then $\operatorname{ndeg}_{F}(\widetilde{B})=4$.

The following proposition is in the spirit of a result due to Wadsworth [12, Theorem 7]. In our case, the notion of norm degree plays an essential rôle.

Proposition 2.5. Let $B=\langle r, s, r s, d\rangle_{b}$ and $C=\langle u, v, u v, d\rangle_{b}$ be two anisotropic bilinear forms of dimension 4 having the same determinant d. Suppose $\operatorname{ndeg}_{F}(\widetilde{B})=$ 8 and $\langle r, s, r s, u, v, u v\rangle$ is isotropic. Then, $B$ and $C$ are similar over $F(\sqrt{d})$ if and only if there exists $x \in F$ such that $\left\langle r, s, r s, d+x^{2}\right\rangle$ is similar to $\left\langle u, v, u v, d+x^{2}\right\rangle$.

Proof. One has $N_{F}(\widetilde{B})=F^{2}(r, s, d)$. Suppose that $\left\langle r, s, r s, d+x^{2}\right\rangle_{b}$ is similar to $\left\langle u, v, u v, d+x^{2}\right\rangle_{b}$ for some $x \in F$. Since $\left(\left\langle d+x^{2}\right\rangle_{b}\right)_{F(\sqrt{d})} \simeq\left(\langle 1\rangle_{b}\right)_{F(\sqrt{d})} \simeq$ $\left(\langle d\rangle_{b}\right)_{F(\sqrt{d})}$, it is clear that the forms $\langle r, s, r s, d\rangle_{b}$ and $\langle u, v, u v, d\rangle_{b}$ are similar over $F(\sqrt{d})$.

Conversely, suppose that $B_{F(\sqrt{d})}$ is similar to $C_{F(\sqrt{d})}$. Then, $\left(\langle\langle r, s\rangle\rangle_{b}\right)_{F(\sqrt{d})}$ is similar to $\left(\langle\langle u, v\rangle\rangle_{b}\right)_{F(\sqrt{d})}$. By the multiplicativity of bilinear Pfister forms, we get that $\left(\langle\langle r, s\rangle\rangle_{b} \perp\langle\langle u, v\rangle\rangle_{b}\right)_{F(\sqrt{d})}$ is metabolic.

(1) If $\langle\langle r, s\rangle\rangle_{b} \perp\langle\langle u, v\rangle\rangle_{b}$ is metabolic, then $\langle\langle r, s\rangle\rangle_{b} \sim\langle\langle u, v\rangle\rangle_{b}$. The forms $\langle\langle r, s\rangle\rangle_{b}$ and $\langle\langle u, v\rangle\rangle_{b}$ are anisotropic since $\langle r, s, r s\rangle_{b}$ and $\langle u, v, u v\rangle_{b}$ are anisotropic. By the uniqueness of the anisotropic part, we get $\langle\langle r, s\rangle\rangle_{b} \simeq\langle\langle u, v\rangle\rangle_{b}$. In particular, $\langle 1\rangle_{b} \perp\langle\langle r, s\rangle\rangle_{b} \simeq\langle 1\rangle_{b} \perp\langle\langle u, v\rangle\rangle_{b}$. Again, it follows from the uniqueness of the anisotropic part that $\langle r, s, r s\rangle_{b} \simeq\langle u, v, u v\rangle_{b}$. Hence, $\langle r, s, r s, d\rangle_{b} \simeq\langle u, v, u v, d\rangle_{b}$.

(2) Suppose that $\langle\langle r, s\rangle\rangle_{b} \perp\langle\langle u, v\rangle\rangle_{b}$ is not metabolic. Since $\langle r, s, r s, u, v, u v\rangle_{b}$ is isotropic, we get $\operatorname{dim}\left(\langle\langle r, s\rangle\rangle_{b} \perp\langle\langle u, v\rangle\rangle_{b}\right)_{\text {an }}=4$. Hence, $\langle r, s, r s\rangle_{b} \simeq\langle a\rangle_{b} \perp \lambda_{1}$ and 
$\langle u, v, u v\rangle_{b} \simeq\langle a\rangle_{b} \perp \lambda_{2}$ for suitable $a \in F^{*}$ and 2-dimensional bilinear forms $\lambda_{1}$ and $\lambda_{2}$ such that $\lambda_{1} \perp \lambda_{2} \simeq\left(\langle\langle r, s\rangle\rangle_{b} \perp\langle\langle u, v\rangle\rangle_{b}\right)_{\text {an }}$. Lemma 2.3 implies that

$$
\lambda_{1} \perp \lambda_{2} \simeq \alpha\left\langle 1, d+x^{2}\right\rangle_{b} \perp \beta\left\langle 1, d+y^{2}\right\rangle_{b}
$$

for suitable $\alpha, \beta, x, y \in F$ with $\alpha, \beta \neq 0$. But by comparing determinants in the last relation, we may suppose that $x=y$. Moreover, by the multiplicativity of bilinear Pfister forms, we may suppose that $\alpha$ is represented by $\lambda_{1}$. Hence, $\langle\langle r, s\rangle\rangle_{b} \simeq$ $\alpha\langle\langle r, s\rangle\rangle_{b}$ since $\lambda_{1}$ is a subform of $\langle\langle r, s\rangle\rangle_{b}$. Consequently, we get

$$
\alpha\left(\langle\langle r, s\rangle\rangle_{b} \perp\left\langle 1, d+x^{2}\right\rangle_{b}\right) \sim\langle\langle u, v\rangle\rangle_{b} \perp \beta\left\langle 1, d+x^{2}\right\rangle_{b} .
$$

The anisotropic part of $\langle\langle r, s\rangle\rangle_{b} \perp\left\langle 1, d+x^{2}\right\rangle_{b}$ has dimension 4 , and thus it is isometric to $\left\langle r, s, r s, d+x^{2}\right\rangle_{b}$; otherwise the form $\langle r, s, r s\rangle_{b}$ would represent $d+x^{2}$, and thus $d \in F^{2}(r, s)$, a contradiction with $\operatorname{ndeg}_{F}(\widetilde{B})=8$. By the uniqueness of the anisotropic part, the form $\langle\langle u, v\rangle\rangle_{b} \perp \beta\left\langle 1, d+x^{2}\right\rangle_{b}$ also has an anisotropic part of dimension 4. It follows from the multiplicativity of bilinear Pfister forms that $\alpha\left\langle r, s, r s, d+x^{2}\right\rangle_{b} \simeq \lambda\left\langle u, v, u v, d+x^{2}\right\rangle_{b}$ for some scalar $\lambda \in F^{*}$. Hence, the claim is established.

We don't know if Proposition 2.5 remains true in the case of norm degree 4. As a corollary of this proposition we get the following:

Corollary 2.6. Let $B$ and $C$ be anisotropic bilinear forms of dimension 4 such that $B \perp C \in I^{3} F$. Then, $B$ is similar to $C$.

Proof. Since $B \perp C \in I^{3} F$, the forms $B$ and $C$ have the same determinant. Set $B=\alpha\langle r, s, r s, l\rangle_{b}$ and $C=\beta\langle u, v, u v, l\rangle_{b}$. Moreover, by Theorem[2.2(1), the forms $B_{F(C)}$ and $C_{F(B)}$ are isotropic. Hence, $N_{F}(\widetilde{B})=N_{F}(\widetilde{C})=F^{2}(r, s, l)$.

(1) Suppose that $l$ is a square. Then, $\alpha\langle\langle r, s\rangle\rangle_{b} \perp \beta\langle\langle u, v\rangle\rangle_{b} \in I^{3} F$. In particular, $\langle\langle r, s\rangle\rangle_{b} \perp\langle\langle u, v\rangle\rangle_{b} \in I^{3} F$. By Theorem 2.2(1), $\langle\langle r, s\rangle\rangle_{b} \sim\langle\langle u, v\rangle\rangle_{b}$. Since $\langle\langle r, s\rangle\rangle_{b}$ and $\langle\langle u, v\rangle\rangle_{b}$ are anisotropic, we get $\langle\langle r, s\rangle\rangle_{b} \simeq\langle\langle u, v\rangle\rangle_{b}$, i.e., $B \simeq \alpha \beta C$.

(2) Suppose that $l$ is not a square. We claim that $\langle r, s, r s, u, v, u v\rangle_{b}$ is isotropic. Indeed, the condition $B \perp C \in I^{3} F$ implies $\alpha\langle\langle r, s\rangle\rangle_{b} \perp \beta\langle\langle u, v\rangle\rangle_{b} \perp \alpha\langle\langle\alpha \beta, l\rangle\rangle_{b} \in$ $I^{3} F$.

(a) If the form $\langle\langle\alpha \beta, l\rangle\rangle_{b}$ is isotropic, then it is metabolic. Hence, $\alpha\langle\langle r, s\rangle\rangle_{b} \perp$ $\beta\langle\langle u, v\rangle\rangle_{b} \in I^{3} F$. By the same argument as in (1), we get $\langle\langle r, s\rangle\rangle_{b} \simeq\langle\langle u, v\rangle\rangle_{b}$, and thus $\langle r, s, r s, u, v, u v\rangle_{b}$ is metabolic.

(b) If the form $\langle\langle\alpha \beta, l\rangle\rangle_{b}$ is anisotropic, then we extend the relation

$$
\alpha\langle\langle r, s\rangle\rangle_{b} \perp \beta\langle\langle u, v\rangle\rangle_{b} \perp \alpha\langle\langle\alpha \beta, l\rangle\rangle_{b} \in I^{3} F
$$

to the field $K:=F\left(\langle\langle\alpha \beta, l\rangle\rangle_{b}\right)$. We get by case (a) that $\langle r, s, r s, u, v, u v\rangle_{b}$ is metabolic over $K$. It follows from [5, Th. 1.2] that $\operatorname{dim}\left(\langle r, s, r s, u, v, u v\rangle_{b}\right)_{\text {an }}$ is a multiple of 4 , which implies that $\langle r, s, r s, u, v, u v\rangle_{b}$ is isotropic. Hence the claim is verified.

Now, we discuss two cases:

(2.1) Suppose that $\operatorname{ndeg}_{F}(\widetilde{B})=8$. Then $l \notin F^{2}(r, s)$, and by Lemma $2.3\langle\langle r, s\rangle\rangle_{b}$ is anisotropic over $F(\sqrt{l})$. Since $B \perp C \in I^{3} F$, we get

$$
\left(\langle\langle r, s\rangle\rangle_{b} \perp\langle\langle u, v\rangle\rangle_{b}\right)_{F(\sqrt{l})} \in I^{3} F(\sqrt{l}),
$$

and by the same argument as in (1) we get that $\left(\langle\langle r, s\rangle\rangle_{b}\right)_{F(\sqrt{l})} \simeq\left(\langle\langle u, v\rangle\rangle_{b}\right)_{F(\sqrt{l})}$. Hence, $B_{F(\sqrt{l})}$ is similar to $C_{F(\sqrt{l})}$. It then follows from Proposition 2.5 that 
$\left\langle r, s, r s, l+x^{2}\right\rangle_{b} \simeq k\left\langle u, v, u v, l+x^{2}\right\rangle_{b}$ for some scalars $x, k \in F$ with $k \neq 0$. In particular,

$$
\langle\langle r, s\rangle\rangle_{b} \sim\left\langle 1, l+x^{2}\right\rangle_{b} \perp k\langle\langle u, v\rangle\rangle_{b} \perp k\left\langle 1, l+x^{2}\right\rangle_{b} .
$$

If we combine this relation with $B \perp C \in I^{3} F$, it is clear that modulo $I^{3} F$ we get

$$
\alpha k\left\langle 1, l+x^{2}\right\rangle_{b} \perp \alpha\left\langle l, l+x^{2}\right\rangle_{b} \perp \beta\langle 1, l\rangle_{b} \in I^{3} F .
$$

Hence

$$
\alpha k\left\langle 1, l+x^{2}\right\rangle_{b} \perp \alpha\left\langle l, l+x^{2}\right\rangle_{b} \sim \beta\langle 1, l\rangle_{b} .
$$

Since $\langle r, s, r s, l\rangle_{b} \sim k\langle\langle u, v\rangle\rangle_{b} \perp k\left\langle 1, l+x^{2}\right\rangle_{b} \perp\left\langle l, l+x^{2}\right\rangle_{b}$, we have $\langle r, s, r s, l\rangle_{b} \sim$ $k\langle\langle u, v\rangle\rangle_{b} \perp \alpha \beta\langle 1, l\rangle_{b}$. By the uniqueness of the anisotropic part and the multiplicativity of bilinear Pfister forms, we conclude that $B \simeq m C$ for some scalar $m \in F^{*}$.

(2.2) Suppose that $\operatorname{ndeg}_{F}(\widetilde{B})=4$. Since $\langle r, s, r s\rangle_{b}$ is anisotropic, the form $\langle\langle r, s\rangle\rangle_{b}$ is also anisotropic, and thus $\left[F^{2}(r, s): F^{2}\right]=4$. Hence, $N_{F}(\widetilde{B})=N_{F}(\widetilde{C})=$ $F^{2}(r, s)=F^{2}(u, v)$ and $l \in F^{2}(r, s)$.

If $B \perp C$ is isotropic, then it is metabolic and thus $B \simeq C$.

If $B \perp C$ is anisotropic, then by Theorem 2.2(1) $B \perp C$ is similar to a 3-fold bilinear Pfister form. Hence, $\operatorname{ndeg}_{F}(\widetilde{B \perp C})=8=\operatorname{ndeg}_{F}(\alpha(\widetilde{B \perp C}))$. By a simple computation using the fact that $l \in F^{2}(r, s)=F^{2}(u, v)$, one gets $N_{F}(\alpha(\widetilde{B \perp C}))=$ $F^{2}(r, s, \alpha \beta)$. Moreover, $\langle\langle r, s\rangle\rangle \simeq\langle\langle u, v\rangle\rangle$ since $F^{2}(r, s)=F^{2}(u, v)$. Hence, $\langle u, v, u v\rangle$ is isotropic over $L:=F(\langle\langle r, s\rangle\rangle)$ since it is a quasi-Pfister neighbor of $\langle\langle u, v\rangle\rangle$. Consequently, $\left(\langle u, v, u v\rangle_{b}\right)_{L} \sim\left(\langle 1\rangle_{b}\right)_{L}$ and similarly $\left(\langle r, s, r s\rangle_{b}\right)_{L} \sim\left(\langle 1\rangle_{b}\right)_{L}$. Hence, after extending the relation $\alpha(B \perp C) \in I^{3} F$ to the field $L$, we conclude that $\left(\langle\langle l, \alpha \beta\rangle\rangle_{b}\right)_{L}$ is metabolic. In particular, $\langle\langle l, \alpha \beta\rangle\rangle_{b} \simeq\langle\langle e, f\rangle\rangle_{b}$ for some $e, f \in$ $F^{2}(r, s)$ [5. Th. 1.2]. This implies that $\alpha \beta \in F^{2}(r, s)$ and thus $\left[F^{2}(r, s, \alpha \beta)\right.$ : $\left.F^{2}\right]=4$, a contradiction.

\section{Proof of Theorem 1.1}

Let $\gamma_{1}, \gamma_{2}$ be two Albert bilinear forms.

(1) (i) If $e^{2}\left(\gamma_{1}\right)$ has length 2 , then it is clear that $i_{W}\left(\gamma_{1}\right)=0$.

(ii) Suppose $e^{2}\left(\gamma_{1}\right)$ has length 1 . Let $k, l \in F^{*}$ be such that $e^{2}\left(\gamma_{1}\right)=\frac{d k}{k} \wedge \frac{d l}{l}$. Let $K$ be the function field of $\tau:=\langle\langle k, l\rangle\rangle_{b}$. Since $\tau_{K}$ is metabolic, one has $e^{2}\left(\gamma_{1}\right)_{K}=0$. It follows from Theorem 2.1 that $\left(\gamma_{1}\right)_{K} \in I^{3} K$, and by Theorem $2.2(1)\left(\gamma_{1}\right)_{K}$ is metabolic. Since $\operatorname{ndeg}_{F}(\widetilde{\tau})=4$ because $\tau$ is an anisotropic 2-fold bilinear Pfister form, it follows from [5, Th. 1.2] that $\gamma_{1}$ is isotropic. Moreover, the form $\gamma_{1}$ is not metabolic by reason of length; hence $i_{W}\left(\gamma_{1}\right)=1$.

(iii) If $e^{2}\left(\gamma_{1}\right)$ has length 0 , then it follows from Theorems 2.1 and 2.2 that $i_{W}\left(\gamma_{1}\right)=3$.

(2) Suppose that $\gamma_{1}$ and $\gamma_{2}$ are anisotropic. We will show that $\gamma_{1}$ is similar to $\gamma_{2}$ if and only if $e^{2}\left(\gamma_{1}\right)=e^{2}\left(\gamma_{2}\right)$.

Suppose that $\gamma_{1}$ is similar to $\gamma_{2}$. Then, $\gamma_{1} \perp \gamma_{2} \in I^{3} F$. It follows from Theorem 2.1 that $e^{2}\left(\gamma_{1}\right)=e^{2}\left(\gamma_{2}\right)$.

Conversely, suppose that $e^{2}\left(\gamma_{1}\right)=e^{2}\left(\gamma_{2}\right)$. By Theorem 2.1, $\gamma_{1} \perp \gamma_{2} \in I^{3} F$. After multiplying, if necessary, $\gamma_{1}$ and $\gamma_{2}$ by suitable scalars, we may suppose that $\gamma_{1} \perp \gamma_{2}$ is isotropic. Hence, by Theorem 2.2(2), the Witt index of $\gamma_{1} \perp \gamma_{2}$ is at least 2. Let $k, l \in F^{*}$ be such that $\gamma_{1} \simeq k\langle 1, l\rangle \perp B$ and $\gamma_{2}=k\langle 1, l\rangle \perp C$ for some 
4-dimensional bilinear forms $B$ and $C$ which have the same determinant $l$. Since $B \perp C \in I^{3} F$, it follows from Corollary 2.6 that $B \simeq m C$ for some scalar $m \in F^{*}$. Since $\gamma_{1} \perp \gamma_{2} \in I^{3} F$, one deduces that $m \gamma_{1} \perp \gamma_{2} \in I^{3} F$ (because $\gamma_{1} \perp m \gamma_{1} \in I^{3} F$ ), i.e., $m k\langle 1, l\rangle \perp k\langle 1, l\rangle \in I^{3} F$. Hence, $m k\langle 1, l\rangle \simeq k\langle 1, l\rangle$ by Theorem $2.2(1)$, and thus $\gamma_{1} \simeq m \gamma_{2}$.

\section{REFERENCES}

[1] R. Baeza, Quadratic forms over semilocal rings, Lect. Notes Math., vol. 655, Berlin, Heidelberg, New York: Springer, 1978. MR0491773 (58:10972)

[2] D. W. Hoffmann, A. Laghribi, Quadratic forms and Pfister neighbors in characteristic 2, Trans. Amer. Math. Soc. 356 (2004) 4019-4053. MR2058517 (2005e:11041)

[3] N. Jacobson, Some applications of Jordan norms to involutorial simple associative algebras, Adv. in Math. 48 (1983) 149-165. MR.700981 (84m:16014)

[4] K. Kato, Symmetric bilinear forms, quadratic forms and Milnor K-theory in characteristic 2, Inventiones Math. 66 (1982) 493-510. MR662605|(83i:10027)

[5] A. Laghribi, Witt kernels of function field extensions in characteristic 2, J. Pure Appl. Algebra 199 (2005) 167-182. MR2134299 (2006c:11041)

[6] A. Laghribi, Sur le déploiement des formes bilinéaires en caractéristiques 2, Pacific J. Math. 232 (2007), 207-232. MR2358037 (2008j:11041)

[7] P. Mammone, D. B. Shapiro, The Albert quadratic form for an algebra of degree four, Proc. Amer. Math. Soc. 105 (1989) 525-530. MR931742 (89g:16029)

[8] A. S. Merkurjev, The norm residue symbol of degree 2 (in Russian), Dokl. Akad. Nauk SSSR 261 (1981) 542-547. English translation: Soviet Math. Doklady 24 (1981) 546-551. MR638926(83h:12015)

[9] J. Milnor, D. Husemoller, Symmetric bilinear forms, New York, Heidelberg: Springer, 1973. MR.0506372 (58:22129)

[10] C.-H. Sah, Symmetric bilinear forms and quadratic forms, J. Algebra 20 (1972) 144-160. MR0294378 (45:3448)

[11] A. Vishik, On the dimensions of quadratic forms, Dokl. Akad. Nauk 373 (2000) 445-447. MR:1788358 (2001h:11042)

[12] A. Wadsworth, Similarity of quadratic forms and isomorphism of their function fields, Trans. Amer. Math. Soc. 208 (1975) 352-358. MR0376527 (51:12702)

Laboratoire de Mathématiques de Lens EA 2462, Faculté des Sciences Jean Perrin, Rue Jean Souvraz - SP18, F-62307 Lens, France

E-mail address: laghribi@euler.univ-artois.fr 